\title{
VALIDAÇÃO EXPERIMENTAL DE SOLUÇÕES DE FLEXIBILIDADE ELÁSTICA APLICÁVEIS A CORPOS DE PROVA C(T) E SE(B)*
}

Gustavo Henrique Bolognesi Donato ${ }^{1}$ João Ferreira de Carvalho Júnior² Régis de Matos Curvelo de Barros ${ }^{2}$

\section{Resumo}

O projeto e a operação de estruturas que podem vir a conter trincas demandam estudos de integridade estrutural suportados por propriedades mecânicas à fadiga e à fratura advindas de ensaios experimentais (p. ex.: curvas $J-R$ ou $d a / d N$ vs. $\Delta K$ ), sendo tais experimentos altamente dependentes da medição instantânea do tamanho da trinca. Essa medição em muitos casos é realizada com base na variação da flexibilidade com o crescimento do defeito. O objetivo do presente trabalho é validar experimentalmente soluções numéricas recentes de flexibilidade elástica que descrevem 0 tamanho instantâneo de uma trinca em corpos de prova de geometria $\mathrm{C}(\mathrm{T})$ e $\mathrm{SE}(\mathrm{B})$ incorporando efeitos tridimensionais. Ainda, objetiva comparar tais soluções com as disponíveis nas normas ASTM vigentes. Corpos de prova $C(T)$ e $S E(B)$ foram usinados conforme as referidas normas e testados em máquina universal de ensaios MTS 810.25. Na sequência, os dados obtidos foram convertidos em valores de flexibilidade normalizada $(\mu)$, determinando-se $o$ tamanho de trinca (a) esperado com base nos referidos equacionamentos. As previsões de tamanho de trinca geradas foram comparadas com medições reais da profundidade de trinca obtidas de fractografias das amostras após separação final. No geral, tanto as soluções da ASTM como de Moreira (2014) foram consideradas acuradas e com erros máximos abaixo de $\sim \pm 1,5 \%$. No entanto, os resultados mostram equivalente aderência experimental das soluções disponíveis nas normas ASTM com as de Moreira (2014) para amostras C(T), mas melhor desempenho das propostas de Moreira para amostras $S E(B)$ de variadas geometrias e profundidades de defeitos.

Palavras-chave: Flexibilidade elástica; Validação experimental; Normas ASTM; C(T); SE(B).

\section{EXPERIMENTAL VALIDATION OF UNLOADING COMPLIANCE SOLUTIONS APPLICABLE TO C(T) AND SE(B) SPECIMENS}

\begin{abstract}
The design and operation of structures that can present cracks require structural integrity studies supported by mechanical properties regarding fatigue and fracture (e.g.: $J-R$ or $d a / d N$ vs. $\Delta K$ curves), which strongly depend on the instantaneous crack size estimation during tests. Such measurement is usually based on the variation of elastic unloading compliance caused by a growing crack. The objective is the experimental validation of recently proposed compliance solutions that describe the instantaneous crack size in $C(T)$ and $S E(B)$ incorporating three dimensional effects. In addition, to compare such solutions to current ASTM standards. $C(T)$ and $S E(B)$ specimens have been machined according to the aforementioned standards and tested in a universal MTS 810.25 test machine. After testing, the acquired results were converted to normalized flexibility $(\mu)$ and thus crack size (a) could be estimated using the recently proposed equations and current standards. Such values could be compared to real fractographic measurements. In general, the results show that both ASTM and proposals from Moreira (2014) are accurate and provide erros below $\pm 1,5 \%$. However, results reveal comparable performance between solutions for $\mathrm{C}(\mathrm{T})$ geometries, while better performance for Moreira's equations for $\mathrm{SE}(\mathrm{B})$ with varying geometries and defect lengths. Keywords: Unloading compliance; Experimental validation; ASTM standards; C(T); SE(B).

1 Engenheiro Mecânico, Doutor, Docente do Departamento de Engenharia Mecânica do Centro Universitário da FEl, São Bernardo do Campo, SP, Brasil, gdonato@fei.edu.br

2 Engenheiro Mecânico, Mestre, Centro Universitário da FEI, São Bernardo do Campo, SP, Brasil.
\end{abstract}




\section{INTRODUÇÃO E MOTIVAÇÃO}

Os desenvolvimentos das últimas décadas conduziram a materiais metálicos de aplicação estrutural com elevadas resistência e tenacidade (por exemplo aços de alta resistência, alumínios de aplicação aeroespacial, entre outros). Em paralelo, esforços de otimização geométrica são crescentes e empregam técnicas numéricas avançadas que resultam em geometrias complexas e que trabalham sob elevados níveis de solicitação, podendo gerar falhas por fratura (usualmente precedidas de extensão dúctil de defeitos) ou pela extensão de trincas por fadiga. Nesse cenário, ensaios de curva- $R$ ( $J$ vs. $\Delta a$ - crescimento dúctil de trinca) e de propagação de trincas por fadiga $(d a / d N$ vs. $\Delta K)$ se tornam de central relevância pois fornecem as propriedades mecânicas de base para muitos protocolos de projeto, previsão de falha e avaliação de integridade estrutural para aceitação de componentes de alta responsabilidade, especialmente em setores como o aeroespacial, óleo e gás e nuclear [1].

O tamanho instântaneo de uma trinca $(a)$ é um dos principais dados de entrada para estimar os parâmetros de carregamento e resistência envolvidos com a fadiga e a fratura [2]. Seja nas aplicações ou em amostras laboratoriais, a medição correta de tal dimensão é de fundamental importância para a determinação das forças motrizes de trinca atuantes do material. Dentre as principais técnicas aplicáveis em laboratório, podem ser destacadas as ópticas, de queda de potencial elétrico e de flexibilidade elástica no descarregamento, sendo o desempenho da última investigado no presente trabalho.

A Figura 1 ilustra a curva $J-R$ de um aço estrutural ferrítico para fins de ilustração e fica claro que a força motriz (no caso integral $J$ ) é apresentada em termos da extensão do defeito $(\Delta a)$. Foi empregada a técnica de flexibilidade elástica no descarregamento para a estimativa do tamanho de trinca, no entanto, o início da curva mostra um crescimento de trinca negativo, o que não é razoável do ponto de vista fenomenológico e possivelmente pode ser atribuído a rotação, plasticidade, entre outros efeitos que fogem do escopo do presente estudo [3]. Assim, fica claro que o estudo das metodologias de estimativa do tamanho instantâneo de defetos merece destaque.

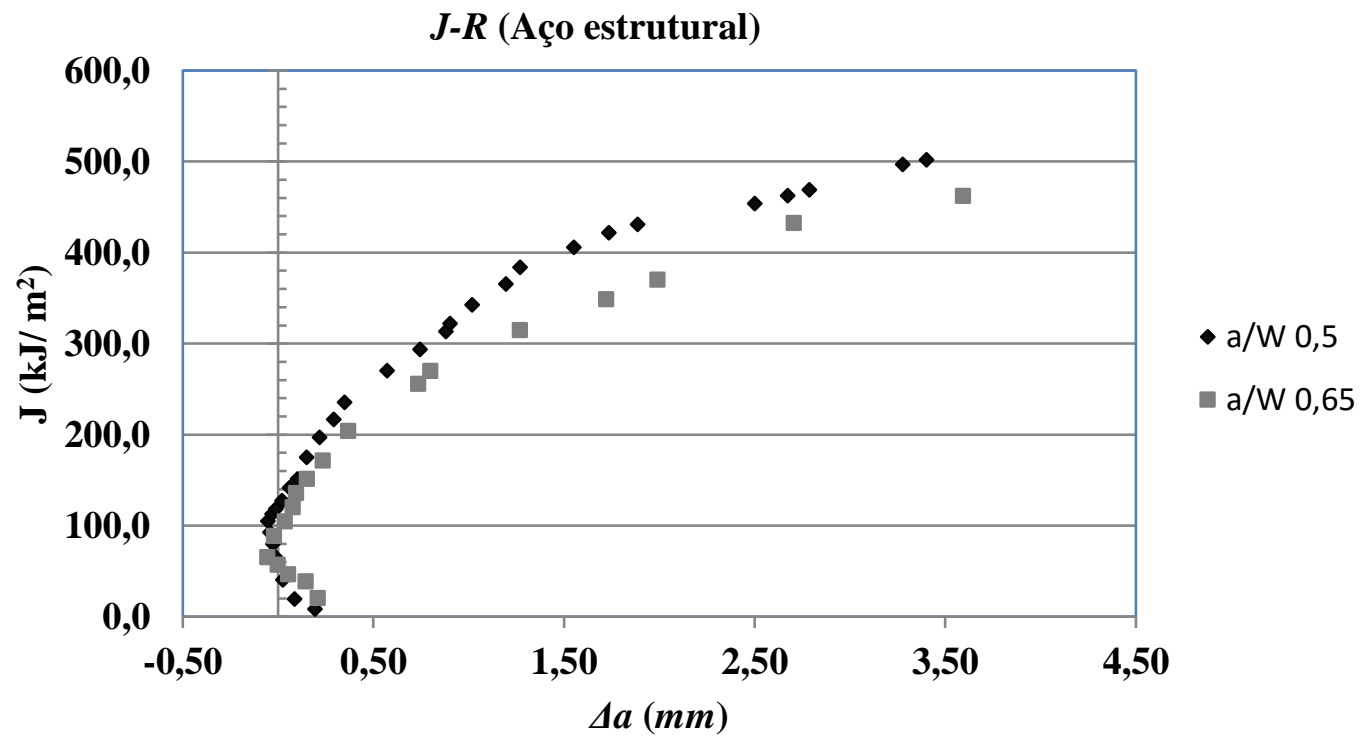

Figura 1: Curva $J-R$ com previsão de crescimento inicial de trinca negativo. $a / W$ representa a profundidade relativa de trinca, sendo $W$ a largura da amostra [3]. 
O principal método aplicado para se medir trincas instantaneamente é a flexibilidade elástica no descarregamento [4-6,8], no qual o corpo de prova sofre diversos descarregamentos (no caso de ensaio monotônico) ou tem seus ciclos avaliados em termos de rigidez momentânea, conforme ilustram respectivamente as Figuras 2(a,b). O objetivo é determinar a rigidez momentânea por meio de uma curva de carga $(P) v s$. deslocamento (nesse caso CMOD - também denotado $V$ - abertura na boca da trinca), podendo-se estimar o tamanho instantâneo da trinca respectiva.

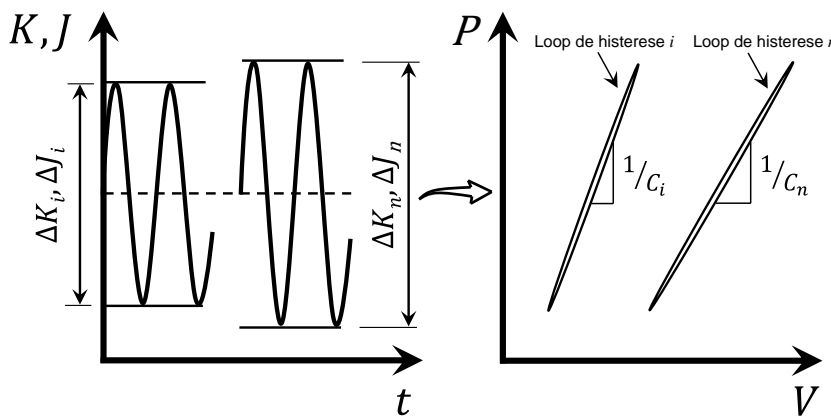

(a)

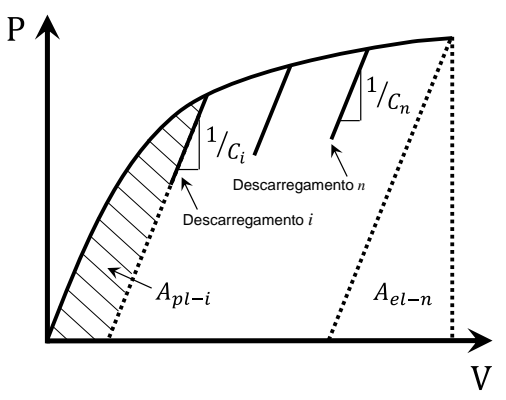

(b)

Figura 2: determinação da rigidez (inverso da flexibilidade) em ensaio ciclico ou monotônico [4]

Para esse fim, existem equacionamentos específicos que relacionam a flexibilidade normalizada $(\mu)$ com o tamanho relativo de trinca $(a / W)$, usualmente por meio de polinômios de $5^{\circ}$ grau. As Equações $(1,2)$ apresentam, respectivamente, as soluções aplicáveis a amostras $\mathrm{C}(\mathrm{T})$ e $\mathrm{SE}(\mathrm{B})$ disponíveis nas normas $\mathrm{ASTM}$ correntes $[4,5]$ considerando CMOD. As dimensões principais podem ser vistas na Figura 5.

$$
\begin{aligned}
\frac{a}{w}= & 1,001-4,6695(\mu)^{1}+18,46(\mu)^{2}-232,82(\mu)^{3}+1214,9(\mu)^{4}-2143,6(\mu)^{5} \\
\mu= & \frac{1}{\left(B_{e f f} \cdot E . C\right)^{0,5}+1} ; B_{\text {eff }}=\mathrm{B}-\frac{\left(B-B_{N}\right)^{2}}{B} \\
\frac{a}{w}= & 0,9997-3,9504(\mu)^{1}+2,9821(\mu)^{2}-3,2141(\mu)^{3}+51,5156(\mu)^{4}-113,031(\mu)^{5} \\
& (0,30 \leq \mathrm{a} \mathrm{W} \leq 1,00) \\
\frac{a}{w}= & 1,0188-4,5367(\mu)^{1}+9,0101(\mu)^{2}-27,333(\mu)^{3}+74,400(\mu)^{4}-71,489(\mu)^{5} \\
& (0,05 \leq \mathrm{a} \mathrm{W} \leq 0,45)
\end{aligned}
$$$$
\mu=\frac{1}{\left[\left(B . W \cdot E^{\prime} \cdot V .4\right) /(P . S)\right]^{0,5}+1}
$$

Embora amplamente empregados pelas normas ASTM E1820 [4] e E647 [5], os equacionamentos correntes acima mencionados são baseados em modelos de elementos finitos que se baseiam em Estado Plano de Tensão (EPT) ou Estado Plano de Deformação (EPD), não levando em conta efeitos tridimensionais e de tunelamento de trincas. Por conta disso Moreira [6] desenvolveu modelos refinados tridimensionais e propôs novos coeficientes de ajuste para os polinômios apresentados que contemplam ao menos os efeitos tridimensionais advindos da espessura e dos estados de tensão em amostras $\mathrm{C}(\mathrm{T})$ e $\mathrm{SE}(\mathrm{B})$. Para fins de ilustração, respectivamente as Equações $(3,4)$ apresentam as soluções de Moreira para amostras de proporções $W=2 B$. As demais soluções podem ser encontradas diretamente no referido trabalho. 
$\frac{a}{w}=0,9239-1,305(\mu)^{1}-25,812(\mu)^{2}+134,687(\mu)^{3}-277,5005(\mu)^{4}+222,363(\mu)^{5}$

$(0,20 \leq \mathrm{a} / \mathrm{W} \leq 0,70)$

$\mu=\frac{1}{\left(B_{e f f} \cdot E . C\right)^{0,5}+1} ; \mathrm{B}_{\mathrm{eff}}=\mathrm{B}-\frac{\left(\mathrm{B}-\mathrm{B}_{\mathrm{N}}\right)^{2}}{\mathrm{~B}}$

$\frac{a}{w}=0,9854-3,45698(\mu)^{1}-1,8995(\mu)^{2}+21,2208(\mu)^{3}-24,8444(\mu)^{4}+5,5067(\mu)^{5}$

$(0,20 \leq \mathrm{a} / \mathrm{W} \leq 0,70)$

$\mu=\frac{1}{\left[\left(B . W \cdot E^{\prime} . V .4\right) /(P . S)\right]^{0,5}+1}$

Moreira [6] demonstrou por meio de verificações numéricas que as equações apresentadas aprimoraram a previsão instantânea do tamanho de trincas para ambas as geometrias. No entanto, o referido trabalho foi exclusivamente numérico, sendo necessária a validação experimental das propostas por meio de ensaios reais. É nesse ponto que se insere o presente trabalho, um primeiro esforço exploratório que objetiva usinar corpos de provas $\mathrm{C}(\mathrm{T})$ e $\mathrm{SE}(\mathrm{B})$ selecionados, estimar seu comprimento de trinca por meio de flexibilidade elástica e comparar com medições físicas reais.

\section{MATERIAIS E MÉTODOS}

As amostras foram usinadas por meio de corte a jato de água a partir de uma chapa de aço ASTM A516 Gr. 70 laminada a quente de espessura $32 \mathrm{~mm}$, na posição T-S (Figura 4a). Após o corte, as amostras sofreram fresamento convencional e a usinagem do entalhe central via eletroerosão a fio (Figura 4b, gerando um raio de ponta inferior a 0,3 mm). As propriedades mecânicas do aço em estudo estão na tabela 1 e foram obtidas da mesma chapa por Ganharul [7].

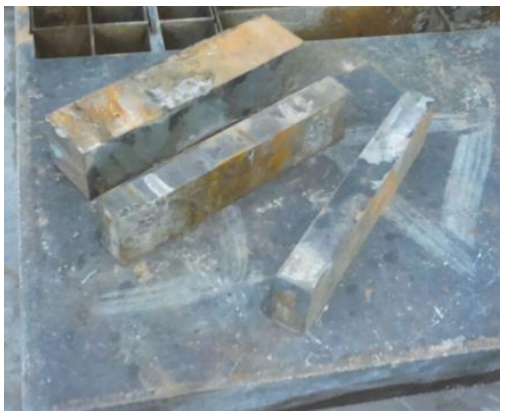

(a)

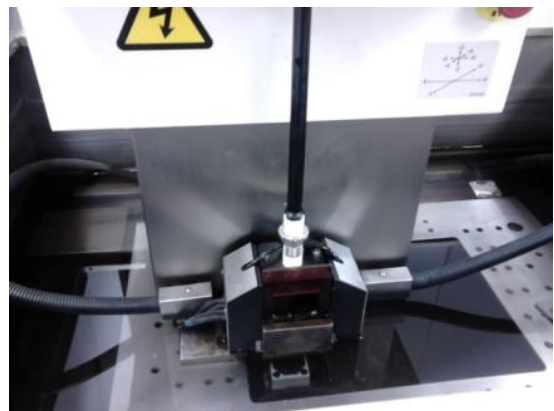

(b)

Figura 4: (a) Corte a jato d'água e (b) eletroerosão a fio.

\begin{tabular}{l|c}
\hline Propriedade mecânica & Valores \\
\hline Módulo de elasticidade longitudinal $(E)$ & $202+/-2,0 \mathrm{GPa}$ \\
\hline Tensão limite de escoamento $\left(S_{L E}\right)$ & $286,5+/-2,1 \mathrm{MPa}$ \\
\hline Tensão verdadeira na fratura $\left(\sigma_{f}\right)$ & $764,5+/-53,1 \mathrm{MPa}$ \\
\hline Coeficiente de resistência $(H)$ & $964,5 \mathrm{MPa}$ \\
\hline Expoente de encruamento & 0,2372 \\
\hline
\end{tabular}

Tabela 1: propriedades mecânicas do aço A516-Gr70 [7]

A geometria básica dos corpos de prova pode ser visualizada na Figura 5. A largura $W$ dos corpos de prova norteou o valor de espessura $B$, sendo que nesse trabalho foram estudadas relações de $W=B, W=2 B$ e $W=4 B$ para ambas as geometrias, tendo 
sido usinados 4 corpos de prova para cada condição de espessura e de profundidade de entalhe/trinca $(a / W=0,2 ; a / W=0,5 ; a / W=0,7)$. A largura $W$ das amostras $S E(B)$ foi mantida constante em 30,5 mm, enquanto para amostras $\mathrm{C}(\mathrm{T})$ em $24 \mathrm{~mm}$. É importante frisar que ambas as geometrias desenvolvidas apresentam dimensões ligeiramente reduzidas em relação ao usual $1 T$ (espessura $B$ de uma polegada e $W=$ $2 B$ ). Tal decisão dificulta um pouco especialmente os ensaios $\mathrm{C}(\mathrm{T})$ (exige maior resolução dos instrumentos e cautela no alinhamento), no entanto, permite a obtenção direta das amostras de diferentes espessuras $B$ direto da chapa por corte a água com reduzido fresamento (vide Figuras 4,5 para compreensão do posicionamento T-S).

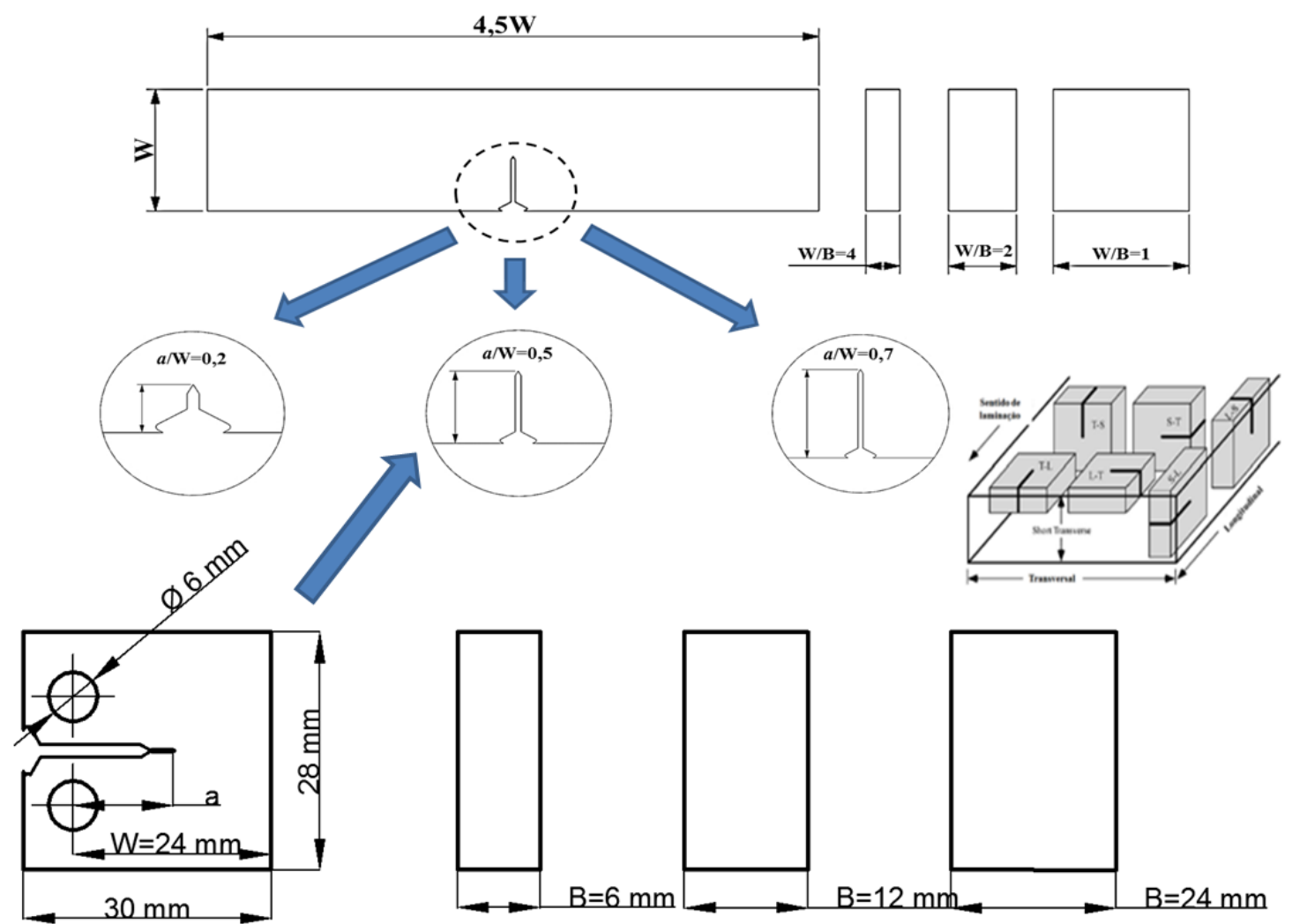

Figura 5: geometria básica dos corpos de prova usinados, obtidos na posição T-S de laminação.

O objetivo na confecção dessas amostras foi prever o tamanho do defeito existente nas amostras avaliando o nível de erro das soluções de flexibilidade. Para tanto, diferentes tamanhos iniciais foram impostos resultando em profundidade relativa $(a / W)$ de $0,2,0,5$ e 0,7. Um total de 24 corpos de prova $S E(B)$ e $24 \mathrm{C}(\mathrm{T})$ foram desenvolvidos. Os testes para mensurar a flexibilidade elástica no descarregamento do espécime seguiram o seguinte protocolo, aquisitando os dados de carga e CMOD em uma máquina de ensaios universal MTS 810.25:

- $\quad$ Carregar a amostra até uma carga a 10\% de $\mathrm{K}_{\operatorname{máx}}\left(18 \mathrm{MPa}^{0} \mathrm{~m}^{0,5}\right)$.

- Taxa de carregamento: $10 \mathrm{~N} / \mathrm{s}$.

- Descarregar até carga nula.

Nessa etapa, uma célula de $2 \mathrm{kN}$ adequadamente calibrada com pesos-padrão foi utilizada para se obter os dados de carga e um clip-gage MTS de curso $3 \mathrm{~mm}$ devidamente calibrado em tambor micrométrico foi empregado na determinação do CMOD. Feito isto, os dados de carga e deslocamento foram tratados em algoritmo MatLab a fim de se obter a flexibilidade normalizada $(\mu)$ e o tamanho do defeito $(a / W)$. O tamanho real do entalhe foi medido por projetor de perfil e por análise fractográfica 
post-mortem. Para o pré-trincamento, as amostras foram excitadas com frequência $10 \mathrm{~Hz}$ e carga correspondente ao valor de $K_{m a ́ x}$, conforme previsto em norma. A Figura 6 ilustra o esquema de fixação e teste de uma das amostras de cada geometria.
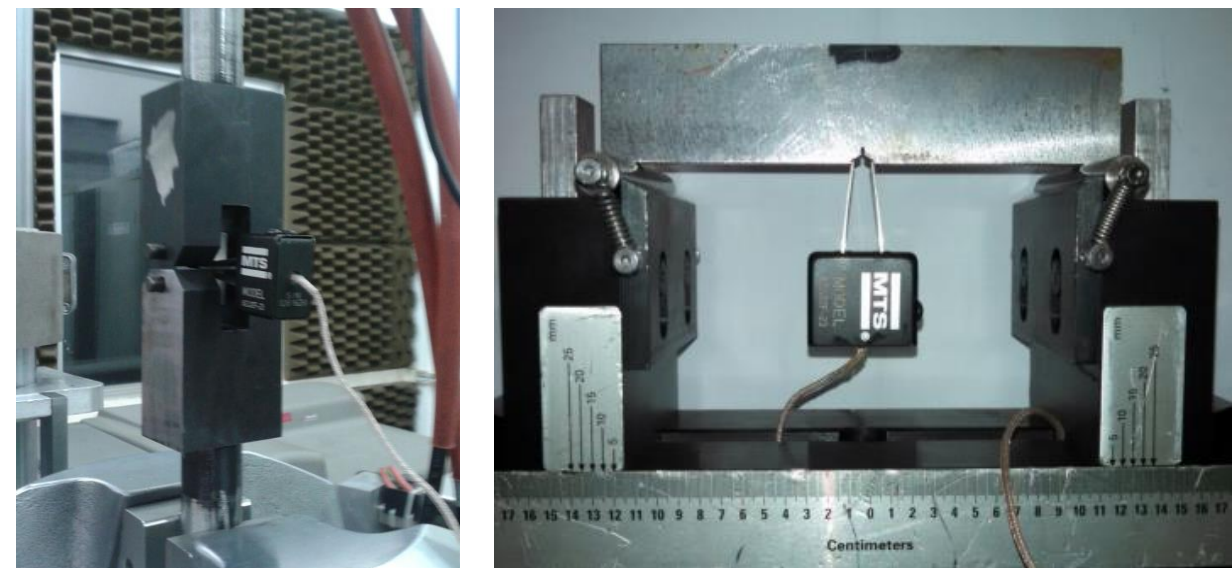

Figura 6: Esquemas de fixação das amostras $C(T)$ e $S E(B)$.

Depois dessa etapa, cada corpo de prova sofreu heat tinting e foi rompido de maneira dúctil para ser possível visualizar o defeito inicial (Figura 7), medido com o auxílio de lupa estereoscópica e um programa analisador de imagem elaborado pelo grupo de pesquisa dos autores. O tamanho médio das pré-trincas de fadiga foi determinado com base nos nove pontos recomendados pela ASTM. No entanto, algumas poucas amostras apresentaram expressivo tunelamento (Figura 7) e foram desprezadas na fase de avaliação das pré-trincas. Ainda, o uso de $10 \%$ de $\mathrm{K}_{\operatorname{máx}}\left(18 \mathrm{MPa}^{0} \mathrm{~m}^{0,5}\right)$ para a avaliação da flexibilidade foi suficiente para a fase de amostra entalhada, mas não para a fase de pré-trinca de fadiga dados os efeitos de fechamento. São aqui apresentados então os resultados para amostras entalhadas e amostras pré-trincadas adicionais seguem em ensaio e são passíveis de ampliação da matriz experimental.

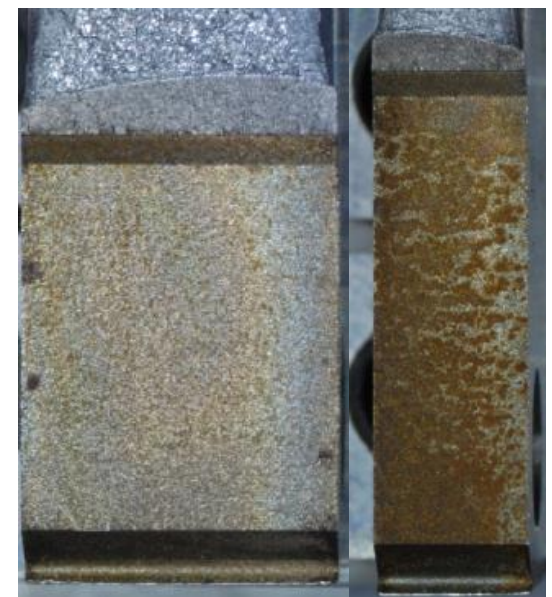

Figura 7: Amostras fraturadas com entalhe retilíneo mas pré-trinca tunelada.

Logo, de posse do tamanho real do entalhe elaborado por eletro-erosão e com reduzido raio de ponta e da estimativa proveniente das equações vigentes e da proposta de Moreira [6], foi possível elaborar as comparações entre as previsões e endereçar as discussões de interesse. 


\section{RESULTADOS E DISCUSSÃO}

No caso da amostra de geometria $\mathrm{C}(\mathrm{T})$, os ensaios envolvendo entalhes rasos $(\mathrm{a} / \mathrm{W} \sim$ $0,2)$ não foram considerados recomendáveis e foram descartados. O entalhe muito raso aproxima a ponta do defeito da linha de carga e faz com que ocorra expressiva deformação local nos corpos de provas próximos dos pinos de carregamento. Assim, existem efeitos de sujeição não negligenciáveis e que apontam para a não recomendação de uso. Já para o caso de entalhes médios $(a / W \sim 0,5)$ e profundos $(a / W \sim 0,7)$, os ensaios foram perfeitamente viáveis e os resultados estão apresentados respectivamente pelas Figuras 8(a) e 8(b). O desvio percentual apresentado pelas figuras é calculado tendo como referência a medição real em lupa estereoscópica.

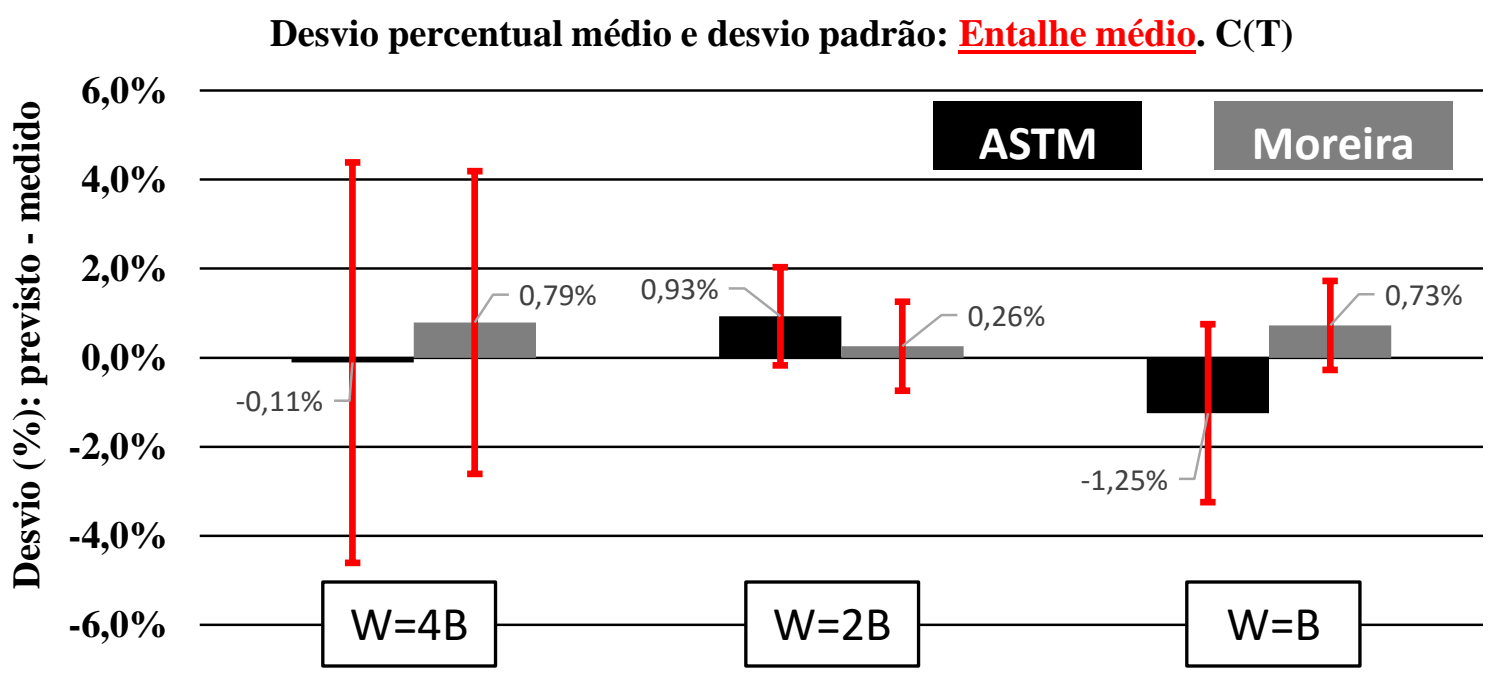

(a)

Desvio percentual médio e desvio padrão: Entalhe fundo. $C(T)$

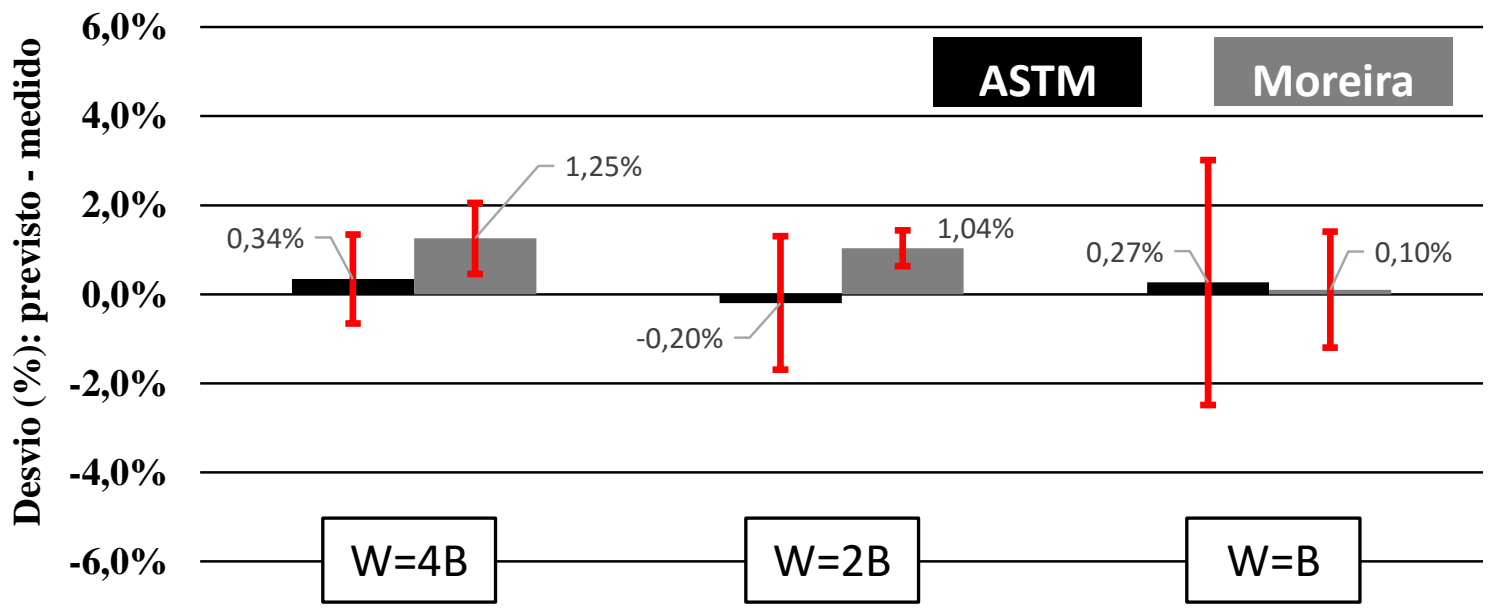

(b)

Figura 8: Desvios percentuais médios para amostras $\mathrm{C}(\mathrm{T})$.

A avaliação da Figura 8 revela que para amostras $C(T)$, a formulações tanto da ASTM como de Moreira apresentam desvios nas previsões bastante reduzidos e no máximo girando em torno de $\pm 1 \%$. Adicionalmente, fica claro que o reduzido número de corpos de prova testados, combinado com um fenômeno que exige expressiva sensibilidade e resolução dos instrumentos, conduziu a desvios-padrão elevados e que não 
permitem uma conclusão objetiva sobre eventuais benefícios ou não no uso das formulações de Moreira [6] em detrimento das disponíveis na ASTM. Mesmo considerando valores médios, em alguns casos a previsão do tamanho instantâneo de trinca era mais acurada pela ASTM, e em outros por Moreira [6]. Ainda, não foi evidenciado um efeito claro da relação $W / B$ no desempenho das propostas em estudo. Embora a avaliação com os dados coletados não possa ser considerada de potencial discriminatório entre as soluções de flexibilidade estudadas para geometrias $\mathrm{C}(\mathrm{T})$, os reduzidos erros das previsões por ambas as soluções permite dizer que as recomendações das normas correntes e também de Moreira [6] estão validadas e apresentam desvios abaixo de $\sim \pm 1 \%$ para entalhes médios e profundos.

Já considerando amostras $\mathrm{SE}(\mathrm{B})$, todas as profundidades relativas de defeito puderam ser ensaiadas sem nenhum contratempo. Não foram evidenciados problemas com o carregamento, tampouco com alinhamento ou deformação anômala da amostra. Os resultados obtidos para a previsão da dimensão dos entalhes estão apresentados nas Figuras 9(a-c) respectivamente para entalhes rasos, médios e profundos.

Primeiramente, pode-se observar na Figura 9 que os desvios nas previsões empregando as soluções da ASTM ficaram dentro de $\sim \pm 1,5 \%$, sendo que curiosamente para $W=2 B$ (proporção mais usual e recomendada pelas normas), os erros foram os maiores chegando na média a $+1,57 \%$ e $-1,21 \%$. Já as previsões empregando as propostas de Moreira [6] apresentaram desvios entre $+0,65 \%$ e $0,81 \%$. Adicionalmente aos valores extremos mais promissores, fica evidente na Figura 9 que considerando o desempenho médio para cada caso as propostas de Moreira sistematicamente forneceram desvios inferiores àqueles obtidos pelas formulações da ASTM.

Embora no caso das amostras $\mathrm{SE}(\mathrm{B})$ também exista sobreposição de diversos desvios-padrão e uma pesquisa com maior quantidade de amostras seja fortemente recomendável, a tendência de maior acurácia das propostas de Moreira [6] é clara e reforçada por alguns casos como de entalhes raso e fundo com $W=2 B$. Por outro lado, embora tal distinção de desempenho esteja presente, os reduzidos erros das previsões por ambas as soluções permite dizer que as recomendações das normas correntes e também de Moreira [6] estão validadas para amostras SE(B) e apresentam desvios médios abaixo de $\sim \pm 1,5 \%$ no caso das normas e $\sim \pm 0,8 \%$ no caso de Moreira [6] para entalhes rasos, médios ou profundos. É altamente recomendada, no entanto, a sequência dos estudos com um maior número de amostras em busca da redução dos desvios-padrão associados aos resultados.

Por fim e analogamente ao observado nas amostras $C(T)$, nas $S E(B)$ não foi evidenciada tendência de aumento ou redução do erro \% com a variação da razão $W / B$. Isto indica que a determinação da flexibilidade normalizada $(\mu)$ surte 0 efeito desejado em permitir que um único polinômio represente a solução de flexibilidade elástica no descarregamento para diferentes espessuras de corpos de prova.

Também deve-se citar que testes de normalidade de Shapiro-Wilk e $t$-student (ambos realizados com auxílio de rotina específica em ambiente MatLab) confirmaram que o tamanho de trinca segue distribuição normal. 
Desvio percentual médio e desvio padrão: Entalhe raso. $\mathrm{SE}(\mathrm{B})$

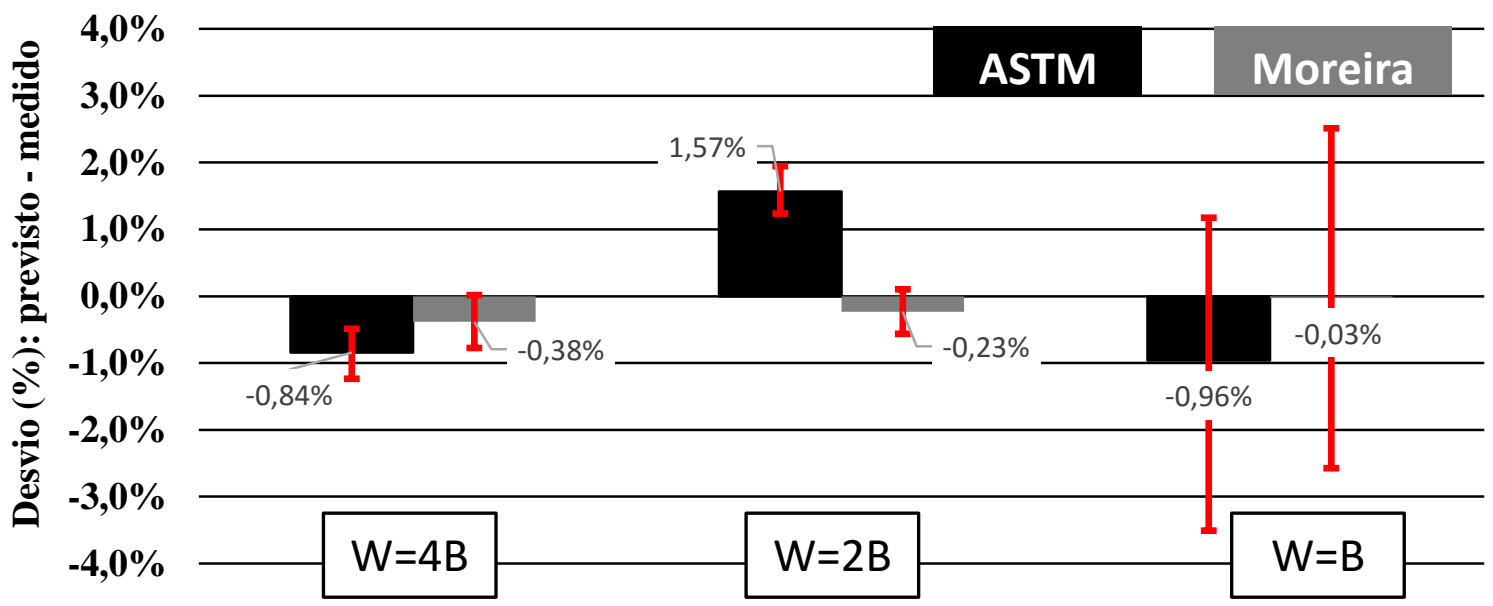

(a)

Desvio percentual médio e desvio padrão: Entalhe médio. SE(B)
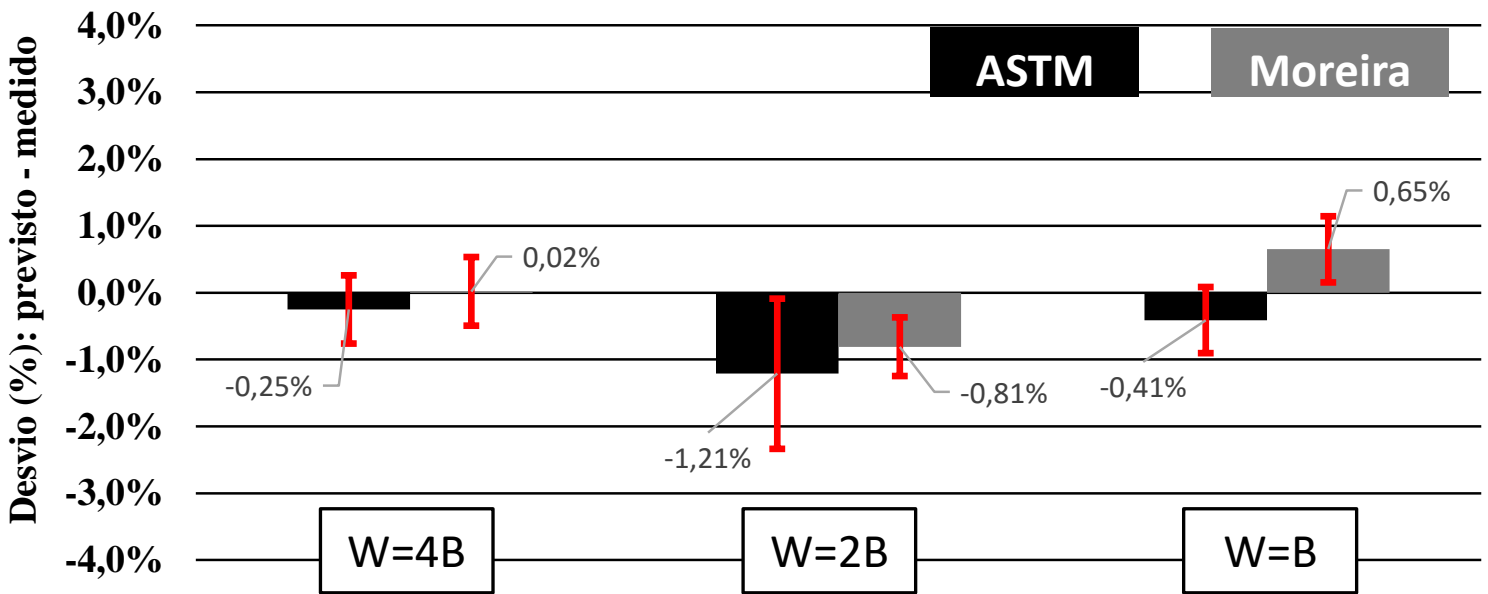

(b)

Desvio percentual médio e desvio padrão: Entalhe fundo. $\mathrm{SE}(\mathrm{B})$

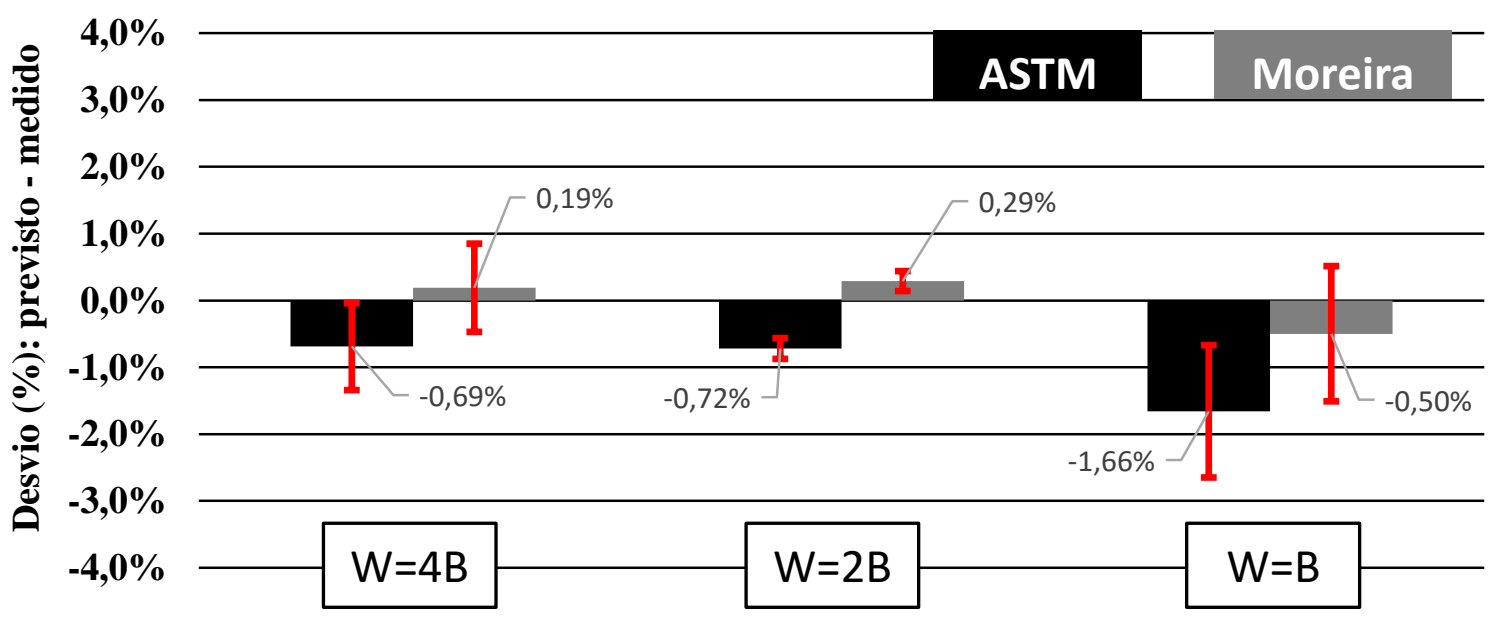

(c)

Figura 9: Desvios percentuais médios para amostras $S E(B)$. 
De acordo com os resultados experimentais, algumas conclusões podem ser endereçadas:

- O ensaio de amostras $\mathrm{C}(\mathrm{T})$ com trincas rasas $(a / W \sim 0,2)$ não é recomendável, já que pode ocorrer deformação local nos furos de carregamento, comprometendo os resultados.

- Nas amostras $\mathrm{C}(\mathrm{T})$, não foi evidenciado efeito de W/B na acurácia das previsões e as formulações tanto da ASTM [4,5] como de Moreira [6] apresentam desvios equivalentes, bastante reduzidos e no máximo girando em torno de $\pm 1 \%$.

- Assim, tanto as soluções disponíveis na ASTM como de Moreira podem ser consideradas validadas e equivalentes em termos de desempenho.

- Nas amostras SE(B), todas as profundidades relativas de defeito puderam ser ensaiadas sem nenhum contratempo. Não foram evidenciados problemas com o carregamento, tampouco com alinhamento ou deformação anômala.

- Ainda nas amostras SE(B), os desvios empregando as soluções da ASTM ficaram dentro de $\sim \pm 1,5 \%$, enquanto empregando as propostas de Moreira [6] apresentaram desvios entre $+0,65 \%$ e $-0,81 \%$. Mesmo com a sobreposição de alguns desviospadrão, existe uma tendência clara de melhor desempenho das propostas de Moreira considerando os desvios médios.

- Em ambas as geometrias não foi evidenciada tendência de aumento ou redução dos desvios com a variação da razão $W / B$. Isto indica que a flexibilidade normalizada $(\mu)$ surte o efeito desejado em permitir que um único polinômio represente a solução de flexibilidade elástica no descarregamento para diferentes espessuras de uma determinada geometria de amostra de fratura.

- Os reduzidos desvios percentuais encontrados não desencoraja o uso dos equacionamentos já consagrados nas normas ASTM, assim como valida o emprego das propostas de Moreira. No entanto, é altamente recomendada a sequência dos estudos com um maior número de amostras em busca da redução dos desvios-padrão associados aos resultados.

\section{Agradecimentos}

Agradecimentos: CAPES pela bolsa concedida ao terceiro autor. Ao CLM-FEI pelo apoio na execução dos ensaios e na confecção dos corpos de prova. Ao Centro Universitário $\mathrm{FEI}$ pela infraestrutura, recursos humanos e recursos materiais para a pesquisa.

\section{REFERÊNCIAS}

[1] ANDERSON, T. L. Fracture mechanics: fundamentals and applications. 3 thrd. ed. Boca Raton, Florida; London: CRC, 2005.

[2] NEWMAN, J.C.,Jr. Stress Analysis of the compact specimen including the effects of pin loading.ASTM STP 560, p. 105-121, 1974.

[3] JOYCE et al.Effects of crack depth and mode of loading on the J-R curve behavior of a high strength steel. ASTM STP 1171: Elastic-Plastic Fracture. Philadelphia, p. 451-468, 1993.

[4] AMERICAN SOCIETY FOR TESTING AND MATERIALS. ASTM E1820: Standard test method for measurement of fracture toughness. Philadelphia, jul. 2013.

[5] __ ASTM E647: standard test method for measurement of fatigue crack growth rates. Philadelphia, jul. 2014. 
[6] MOREIRA, F. C., DONATO, G. H. B. Effects of side-grooves and 3D geometries on compliance solutions and crack size estimations applicable to $\mathrm{C}(\mathrm{T}), \mathrm{SE}(\mathrm{B})$ and clamped SE(T) specimens, proc. of ASME PVP 2013, 2010.

[7] GANHARUL, G.K. Experimental analysis of the effect of triaxiality post- instability tensions in determining the stress-strain properties through uniaxial tensile test . 2012.157 f .

Dissertation (Master of Engineering) - University Center of FEI , São Bernardo do Campo, 2012.

[8] JABLONSKI, D. A. et al. Compliance functions for various fracture mechanics specimens. Engineering Fracture Mechanics. v. 22, p. 819-827, 1985.

[9] VESTRAETE et al., Evaluation and interpretation of ductile crack extension in SENT specimens using unloading compliance technique. Engineering fracture mechanics. P. 190203, nov. 2013.

[10] ELBER, W. Fatigue Crack Closure under Cyclic Tension. Engineering Fracture Mechanics. v. 2, p. 37-45, 1970.

[11] RUGGIERI, Claudio. Further results in $\mathrm{J}$ and CTOD estimation procedures for SE(T) fracture specimens - Part I: Homogeneous materials. Engineering fracture mechanics, $p$. 245-265, nov. 2011. 